\title{
Longitudinal interplay between peer likeability and youth's adaptation and psychological well-being: A study of immigrant and nonimmigrant adolescents in the school context
}

\author{
Frosso Motti-Stefanidi, ' $\odot$ Vassilis Pavlopoulos,' \\ Stefanos Mastrotheodoros, ${ }^{2}$ and Jens B. Asendorpf ${ }^{3}$
}

\begin{abstract}
The present study examined the direction of effects between peer likeability and youth's school adjustment and psychological well-being, and the moderation of these effects by students' immigrant status. One thousand one hundred and eighteen students (63\% immigrants) nested in 57 Greek middle-school classrooms took part in the study (Wave I; age $M=12.6$ years). Data were collected from multiple sources and informants. The results reveal complex, in some cases bidirectional effects over time between peer likeability and different indices of school adjustment and psychological well-being. Being liked by Greek, but not by immigrant, classmates influenced students' wellbeing over time. In contrast, being liked by either immigrant or Greek classmates predicted changes in students' school adjustment. The results highlight the importance of supporting positive peer relations among youth in order to promote other adaptation outcomes. In the case of immigrant youth, they suggest that interventions need to promote positive intergroup contact between them and their nonimmigrant classmates in order to support their well-being.
\end{abstract}

\section{Keywords}

Peer likeability, immigrants, school adjustment, psychological well-being, longitudinal, classroom

Being liked and not being disliked by peers is a key developmental task for youth and a sign of positive adaptation (Masten, 2014). Schools are an important context for the development of peer relations. The quality of peer relations in the school context has wide ranging consequences for their school adjustment and psychological well-being. Problematic peer relations are prospectively linked to several forms of behavioral and affective maladjustment (Prinstein et al., 2018).

For immigrant youth, schools are also the main acculturative context where they are exposed to the host culture. Positive peer relations at school are both a developmental and an acculturative task (Motti-Stefanidi, Berry, Chryssochoou, Sam, \& Phinney, 2012; Suarez-Orozco, Motti-Stefanidi, Marks, \& Katsiaficas, 2018). Like all youth, immigrant youth need to be liked and accepted by their peers, independently of the immigrant status or the ethnicity of these peers. From an acculturation perspective, they must also learn how to navigate successfully between at least two different cultures and be accepted by both intra- and interethnic peers. Whether a young immigrant is well adapted with respect to peer relations rests on both of these criteria (Suárez-Orozco et al., 2018). Success with respect to these developmental and acculturative tasks can have significant positive consequences for their sense of belonging and adjustment in school (Motti-Stefanidi \& Masten, 2017). In contrast, experiences of rejection and discrimination by peers can have negative consequences for their school adjustment and well-being (Marks et al., 2015) as well as for their acculturation into the host culture, which tend to persist over time (Motti-Stefanidi, Berry, Chryssochoou, Sam, \& Phinney, 2012).
The purpose of this study is two-fold. The first goal is to examine whether and how youth's likeability among classmates is longitudinally linked to their school adjustment and well-being. Peer preferences were used as an index of youth's general likeableness (Rubin et al., 2015). Peer preferences refer to the difference between peer-nominated acceptance and rejection standardized within classroom. Positive peer preferences suggest that youth are more accepted by their peers than rejected. Negative peer preferences indicate the opposite. The first goal concerns all youth, independently of immigrant status. The second goal is to examine whether and how these longitudinal links are moderated by immigrant status.

The present article is based on data from a larger longitudinal investigation, the Athena Studies of Resilient Adaptation (AStRA) project, conducted in Greece. Data were collected from schools in socially disadvantaged neighborhoods of Athens with a high proportion of immigrants. Immigrant youth are defined as those who have at least one foreign-born parent. This group includes both

\footnotetext{
I National and Kapodistrian University of Athens, Greece

2 Utrecht University, The Netherlands

${ }^{3}$ Humboldt University Berlin, Germany
}

\section{Corresponding author:}

Frosso Motti-Stefanidi, School of Philosophy, National and Kapodistrian University of Athens, Athens, 15784, Greece.

Email: frmotti@psych.uoa.gr 
first-generation immigrants who were born outside the host country and second-generation immigrants who were born within the host country (Suarez-Orozco et al., 2018). The project included Greek and immigrant students nested in the same classrooms. Participants were followed for 3 consecutive years through middle school.

The study contributes to the extant literature on both immigrant and nonimmigrant youth adaptation with respect to peer relations in a number of ways. First, data on a large number of adaptation indices were collected with multiple methods, and from multiple informants. Second, all students in each classroom served as the reference group in the sociometric test. The peer data are disaggregated by immigrant status, allowing the examination of the way peer likeability and adaptation are longitudinally linked when the evaluators in the sociometric test, as well as those being evaluated, are Greeks or immigrants. Third, it tests these effects while controlling for continuity in a given construct over time. To the best of our knowledge, no other study on the link between peer likeability and a significant number of indices of the school adjustment and well-being of youth, in general, and of immigrant youth, in particular, includes these parameters.

Participants were early adolescents. During this developmental period, adolescents spend less time at home with parents and more time with peers in school and in the community. In spite of a continued closeness to their parents, they put a greater stock in the expectations and opinions of their peers (Sroufe et al., 2005). Given the salience of peers in adolescents' life, it becomes a particularly fruitful period to examine, first, whether and how peer relations affect and are affected by youth's school adjustment and wellbeing, and, second, whether and how immigrant versus nonimmigrant peers affect the school adjustment and well-being of their classmates.

\section{Links Between Peer Preferences and Youth's Adaptation and Well-being}

The AStRA project was framed within an integrative resilience developmental framework for understanding individual differences in the adaptation of immigrant youth (Motti-Stefanidi et al., 2012; Suárez-Orozco et al., 2018). The key question addressed is "who among immigrant youth adapt well and why?" The quality of their adaptation is judged based on how well they do with respect to (a) developmental and (b) acculturative tasks, as well as, (c) based on their psychological well-being. A guiding principle of this resilience conceptual framework (Suarez-Orozco et al., 2018), which is grounded on developmental systems theory (Bronfenbrenner \& Morris, 2006), is that adaptation is a dynamic process which is shaped by the current interplay between risks and resources as well as by youth's history of prior successes and failures in key developmental (Cicchetti \& Rogosh, 2002) and acculturative (Motti-Stefanidi, 2018) tasks. Thus, adaptation is shaped by many interactions and the directions of influence are expected to be reciprocal rather than unidirectional (Masten \& Kalstabbaken, 2018).

Guided by this conceptual model, the present study examines the longitudinal interplay between, on the one hand, peer likeability, which is both a developmental task and a context for youth's adaptation, and, on the other, their school adjustment and wellbeing. First, the study examines whether positive and negative experiences in the peer context influence over time youth's school adjustment and well-being. The extant literature suggests that being accepted or rejected by peers is consequential for a number of adaptation domains (Prinstein et al., 2018; Ryan \& Shin, 2018). Second, the study also focuses on the role of adaptive history for youth's current adaptation with respect to peer relations. Thus, the quality of youth's school adjustment, which is a key developmental task, and their well-being are expected to have longitudinal effects on whether they are liked by peers (Rubin et al., 2015).

The peer relations literature provides partial support to the key expectation that the direction of influence between peer likeability and adaptation will be reciprocal. Next, we review this literature including all three indices of peer likeability (acceptance, rejection, peer preferences), since most studies examine separately acceptance and rejection (see Prinstein et al., 2018).

The North American peer relations literature mostly focuses on the link between peer rejection and aggression (including disruptiveness, physical aggression, and negative behavior) and provides strong support to the prediction that this link is bidirectional (Rubin et al., 2015). Negative peer preferences are both a predictor of future aggression and a consequence of youth's aggressive behavior (e.g., Lansford et al., 2010). However, studies conducted outside North America have yielded mixed results. For example, one study of preadolescents conducted in Taipei shows that physical aggression is longitudinally related to low likeability, whereas peer acceptance and rejection did not predict over time changes in physical aggression (Tseng et al., 2013). In contrast, the results of a study conducted with preadolescents in Finland found that peer rejection predicted over time increases in bullying, a subtype of aggression, but not vice versa (Sentse et al., 2015).

Peer rejection has also been shown to be linked to increases, whereas peer support to decreases, in symptoms of depression (Finan et al., 2018; Prinstein et al., 2018). However, there is also some evidence in favor of the opposite direction of effects. For example, in one study of primary school-age children symptoms of depression predicted more negative and fewer positive sociometric nominations (Kennedy et al., 1989), suggesting that depressed peers are less likeable compared to nondepressed peers.

According to the Social Information Processing (SIP) model (Dodge \& Crick, 1990), the longitudinal link between peer rejection and both aggression and depression may be explained by the way some youth interpret particular events, which influences the way they will respond. Youth who believe that other people behaved in a certain situation with hostile intent are more likely to respond aggressively than are children who believe that other people behaved with benign intent or that a negative interaction occurred by accident. This aggressive reaction creates a vicious circle, further increasing future rejection by peers and exacerbating SIP problems (Lansford et al., 2010). In this line, it has been argued that peer rejection may lead some children to attribute their social failures to internal causes (Rubin et al., 2009). Self-blame may lead to depression, low self-esteem and withdrawal (Nolen-Hoeksema et al., 1992).

Acceptance and rejection by peers are also linked longitudinally to self-esteem. Reitz et al. (2016) found, based on data from a previous cohort of the AStRA studies, that peer preferences predicted longitudinally changes in self-esteem, but not vice versa. This finding, which is in accordance with sociometer theory, suggests that self-esteem serves as an internal gauge of others' evaluations of the individual (Leary \& Baumeister, 2000).

Finally, extant evidence suggests that peer likeability is linked to academic achievement and motivation (Ryan \& Shin, 2018). Higher likeability by peers leads over time to increases in youth's 
academic achievement and motivation. One study found a bidirectional effect whereby higher academic achievement predicted being liked by peers, which in turn predicted higher academic achievement (Veronneau et al., 2010). However, the latter path seemed to be less consistent. How can these links be explained? Better liked students tend to have positive and productive interactions with their classmates and going to school seems to be for them a rewarding experience, both academically and socially (Ryan \& Shin, 2018). In contrast, rejected students, who may have issues with aggression, often focus on their conflicts with peers rather than on their studies, or instead they may become withdrawn and worry more about what others think of them rather than focusing on their academic work.

\section{Moderation by Immigrant Status}

In the multicultural classrooms of this study, the question arises whether being liked by Greek or immigrant peers equally affects the school adjustment and well-being of students. To the best of our knowledge, the effect of immigrant status on the link between peer likeability and youth's adaptation has not been tested by researchers.

In multiethnic societies, ethnic majority members are considered to have a higher societal standing compared to ethnic minority members (Verkuyten et al., 1996). Since immigrants are a minority in host countries, their societal status is expectedly lower.

Societal status may be particularly relevant in the study of immigrant youth peer relations. For example, Asendorpf and MottiStefanidi (2017) found, based on data from the AStRA project, that Greek students, compared to immigrant students, were more accepted (and less rejected) by their classmates. Only in classrooms with at least two thirds of immigrants, the societal status advantage of Greeks was compensated and immigrant students were more liked by their classmates. Similar findings were reported by Jackson et al. (2006) who studied peer likeability among African and European American middle school students. Thus, majority youth who have a higher societal status also tend to have a higher social status in their classrooms.

Extant research suggests that high status groups in elementary and middle school classrooms are more powerful agents of socialization than low status groups (Ellis \& Zarbatany, 2007). In this line, better liked youth have been shown to have more influence over their peers' behavior compared to less liked youth (Laursen, 2018). Together these findings suggest that nonimmigrant students, who are more accepted by their peers, will have greater influence over their classmates' behavior, compared to their immigrant counterparts, particularly in classrooms with fewer immigrants.

\section{The Present Study}

According to an OECD (2016) report, $60 \%$ of Greek citizens indicate that they view immigrants unfavorably, and more than two thirds of the Greek population believes that immigrants do not contribute to the country's collective well-being. According to a joint OECD (2015) and EU report, 35\% of the immigrants in Greece report feeling discriminated against which ranks Greece first in terms of immigrants' perceived discrimination in Europe.

Immigrant youth refer in this article to youth who either migrated themselves (first generation) or who were born in the host country to immigrant parents (second generation). Immigrants include students representing the two largest immigrant groups in
Greece (Pavlopoulos \& Motti-Stefanidi, 2017). One group consists of immigrants of the diaspora called Pontian-Greeks. They retained their Greek culture for many centuries, but never lived in Greece before migrating. Their language, which is a dialect rooted in Ancient Greek, is incomprehensible to modern Greeks. Although the Greek government accorded them full citizenship status, native Greeks refer to Pontian-Greeks as the "Russians" and do not view them as "real Greeks." In contrast, immigrants from Albania, who at first entered the country as undocumented economic immigrants, were considered guest workers. The remaining immigrants came mostly from other Eastern European countries such as Bulgaria, Romania, or former states of the Soviet Union such as Russia or Moldavia.

Although these immigrant groups differ in numerous ways, they also share a number of commonalities (Pavlopoulos \& MottiStefanidi, 2017; Triandafyllidou, 2014). First, in all cases either they or their parents were not born in Greece; that is, all faced the challenges of acculturation and the need to learn how to navigate between at least two cultures. Second, they all came from countries with unstable and poor economic situations to a country relatively more affluent. As a result, most perceive their new situation as a vast economic improvement. Third, they all have to face similar economic and social difficulties in their adaptation to the same host country. They all experience significant, even though not the same degree of, discrimination.

\section{Hypotheses}

Based on the literature reviewed, we formulate the following four hypotheses.

The first hypothesis is that peer preferences and conduct are bidirectionally linked. This hypothesis is based on solid, conceptually driven evidence showing that being preferred by peers leads to better conduct, and better conduct leads to being preferred by peers (e.g., Lansford et al., 2010; also see Prinstein et al., 2018). Two studies conducted outside the U.S., which yielded contradictory results, focused on specific, narrowly defined, types of aggression, namely bullying (Sentse et al., 2015) and physical aggression (Tseng et al., 2013). Our measure, as well as the Lansford and colleagues' measures, was broader including, in addition to physical aggression and bullying, disruptiveness and negative behaviors.

The second hypothesis is that being preferred by peers will over time lead to decreases in symptoms of depression (see Prinstein et al., 2018), increases in self-esteem (Reitz et al., 2016) and school engagement (see Ryan \& Shin, 2018). The test for the opposite direction from the latter to peer preferences is exploratory. There is some evidence regarding the opposite direction from depressive symptoms to peer preferences but it is scant and focused on younger children whereas the present study focuses on adolescents.

The third hypothesis is that academic achievement will predict over time changes in peer preferences but not the other way around. Even though extant evidence leads to the expectation of a bidirectional link between academic achievement and peer preferences, our own data have shown a very high stability of academic achievement over the three years of middle school (e.g., Motti-Stefanidi, Asendorpf, \& Masten, 2012). As a result, little variance in academic achievement is expected over the middle school years that could be explained by peer preferences.

The fourth hypothesis is that being preferred by the higher status Greek peers is more important for students' school adjustment and 
well-being than being preferred by immigrant students. This hypothesis is based on the argument that the societal status of immigrants in Greece is lower since they are a clear minority (Pavlopoulos \& Motti-Stefanidi, 2017) and, relatedly, on the observation that Greek students are more liked (and less disliked) compared to immigrant students (Asendorpf \& Motti-Stefanidi, 2017). As a result, they are expected to have more influence over their peers' behavior.

Finally, we also explored whether the cross-lagged effects between, on the one hand, peer preferences and, on the other, school adjustment and well-being were different for Greek versus immigrant nominees.

\section{Method}

\section{Sample}

The initial sample consisted of adolescent students living in neighborhoods with a high proportion of immigrant families in Athens, Greece, that were assessed after the first trimester in secondary school amidst a major economic crisis (early in 2013). Assessed were 1,118 students who attended 57 secondary-school grade 1 classes in 14 public schools (age $M=12.6$ years, $S D=0.57$; $53 \%$ male). Of these students, $63 \%$ were immigrants (22\% first generation, who were born in a country other than Greece, $78 \%$ second generation, who were born in Greece); first-generation immigrants had spent $60 \%$ (range 1-99\%) of their lifetime in Greece. More than $90 \%$ of the immigrant students' parents came originally from Eastern Europe, particularly from Albania and expatriates from the former Soviet Union (called Pontic-Greeks). The sample was reassessed 1 year later (attrition rate $8 \%$ ) and 2 years later (additional attrition rate also $8 \%$ ).

\section{Measures}

Immigrant status. This was assessed in terms of the ethnicity of students' mother and father. Immigrant youth are those who have at least one foreign-born parent. Greek students are defined as having both parents being Greek (see Suarez-Orozco et al., 2018).

Peer nominations. A sociometric procedure was used to measure peer-perceived likeability in the classrooms. It is considered the gold standard for assessing adolescent likeability (Cillessen \& Marks, 2011). In each wave, all students in each classroom were asked to write down the names of up to three classmates that they liked most and three classmates they liked least; the ethnicity of each nominator was recorded in terms of being of Greek, Albanian, Pontian, or other ethnicity. In order to control for the number of nominating classmates, we separately computed for each student the percentage of all, only Greek, or only immigrant nominating classmates that positively nominated him or her $\%$ received like most nominations); likewise, we computed $\%$ received like least nominations separately for all, Greek, and immigrant nominators. These percentage scores measure a student's acceptance or rejection by all classmates, by Greek classmates, and by immigrant classmates. The six scores could range from $0 \%$ to $100 \%$. Finally, we computed for each student a social preference score by taking the difference of $\%$ received liked most and $\%$ received liked least nominations. Jiang and Cillessen (2005) reported in their metaanalysis of research with social preference scores a good average short-term test-retest reliability of .82 and concluded that these scores are particularly useful for longitudinal studies of adolescents.

The following measures of well-being and adaptation were assessed in all waves with identical items.

Self-esteem. Self-esteem was self-rated by the students on the 10item Rosenberg Self-Esteem Scale (Rosenberg, 1965). The scale demonstrated good reliability and validity for adolescents and different ethnic groups (Robins et al., 2001). Adolescents rated their agreement to items (e.g., "On the whole I am satisfied with myself") on a 5-point scale ( $1=$ strongly disagree to $5=$ strongly agree). The internal consistencies of the scale were sufficient ( $\alpha \mathrm{s}$ above .77 for both immigrants and Greeks in all three waves).

Depressive symptoms. These were self-rated by the students on 10 items of the Depression Scale (DEPS) by Salokangas et al. (1995), each rated on a 4-point scale not at all-a lot. The internal consistencies of the scale were high ( $\alpha$ s above .81 for both immigrants and Greeks in all three waves).

Conduct. Greek language teachers rated the disruptiveness of each student in the classroom on 6 items, each rated on a 5-point scale, ranging from not at all (1) to very much (5). The items assessed the degree to which the student was aggressive towards peers, disruptive in class, or exhibited negative conduct. The 6 items were the following: "makes fun of other kids in class," "gets involved in fights," "keeps company with kids that create problems," "is disruptive during class," "often lies or is deceitful," "steals from the school or elsewhere." All items were related to externalizing problems. The items were reversely coded such that high scores indicate good conduct. The internal consistencies of the scale were high ( $\alpha \mathrm{s}$ above .90 for both immigrants and Greeks in all three waves).

School engagement. The same teachers who rated conduct also rated the engagement of each student in the classroom on 4 items, each rated on a 5-point scale, ranging from "not at all" to "very much." These items assessed the degree to which the student is motivated and engaged in schoolwork, namely "concentrates in class," "participates in class," "is studying," "is cooperative." The scale had a high internal consistency ( $\alpha$ s above .90 for both immigrants and Greeks in all three waves).

Academic performance. Performance was assessed in terms of a student's grade point average (GPA) for the first trimester of each school year obtained from school records. These time points closely corresponded to the times of assessing the other variables in the classrooms. Grade points in Greek middle schools are rated by teachers on a 20-point scale, with higher points indicating better performance. The GPA of each student was based on the judgments of at least four different teachers for five different subjects during the first trimester of each school year of data collection that were highly consistent across subjects within each wave ( $\alpha$ s above .95). GPA consisted of the average across all subjects on the 20-point scale.

\section{Procedure}

The study was approved by the Ministry of Education and school principals. Students' parents signed a consent form stating that their child was allowed to take part in the study. More than $95 \%$ of the parents gave consent. Participants were tested in their classrooms (group testing) on two different days of the second trimester of each 
Table I. Means, standard deviations and initial intercorrelations of the main variables.

\begin{tabular}{|c|c|c|c|c|c|c|c|c|c|c|c|c|c|c|c|c|c|}
\hline \multirow[b]{2}{*}{ Variables (ranges) } & & \multicolumn{3}{|c|}{ TI } & \multicolumn{3}{|c|}{$\mathrm{T} 2$} & \multicolumn{3}{|c|}{ T3 } & \multicolumn{7}{|c|}{ Initial intercorrelations } \\
\hline & & $N$ & $M$ & $S D$ & $N$ & $M$ & $S D$ & $N$ & $M$ & $S D$ & PGR & PIM & SES & DEP & CON & ENG & GPA \\
\hline Preference total $(-2,2)$ & PTO & 1,046 & 0.01 & 0.21 & 905 & 0.02 & 0.20 & 902 & 0.02 & 0.20 & $.69 * * *$ & $.86 * * *$ & $.14 * * *$ & -.05 & $.24 * * *$ & $.31 * * *$ & $.36 * * *$ \\
\hline By Greeks & PGR & 1,028 & 0.02 & 0.33 & 890 & 0.03 & 0.30 & 828 & 0.03 & 0.27 & & $.34 * * *$ & $.08 * *$ & -.04 & $.20 * * *$ & $.28^{* * * *}$ & $.30 * * *$ \\
\hline By immigrants & PIM & 1,046 & 0.02 & 0.22 & 905 & 0.02 & 0.23 & 842 & 0.03 & 0.23 & & & $.13 * * *$ & -.05 & $.18 * * *$ & $.22 * * *$ & $.27^{* * * *}$ \\
\hline Self-esteem (I-5) & SES & 1,030 & 3.70 & 0.80 & 937 & 3.52 & 0.86 & 852 & 3.60 & 0.85 & & & & $-.50 * * *$ & $.10 * *$ & $.20 * * *$ & $.25 * * *$ \\
\hline Depression (I-5) & DEP & 1,029 & 1.85 & 0.58 & 937 & 1.88 & 0.63 & 852 & 1.95 & 0.63 & & & & & -.02 & $-.10 * * *$ & $-.14^{* * *}$ \\
\hline Conduct $(I-5)$ & $\mathrm{CON}$ & $\mathrm{I}, 078$ & 4.34 & 0.88 & 966 & 4.47 & 0.69 & 931 & 4.51 & 0.65 & & & & & & $.57^{* * * *}$ & $-.37^{* * * *}$ \\
\hline $\begin{array}{l}\text { School engagement } \\
\qquad(I-5)\end{array}$ & ENG & I,078 & 3.43 & 1.18 & 966 & 3.47 & 1.10 & 931 & 3.37 & 1.09 & & & & & & & $.75^{* * * *}$ \\
\hline $\begin{array}{l}\text { Grade point average } \\
\qquad(I-20)\end{array}$ & GPA & 1,115 & 14.42 & 2.99 & 987 & 14.54 & 2.83 & 939 & 14.36 & 2.83 & & & & & & & \\
\hline
\end{tabular}

Note. Initial sample size $N=1,1$ I8. TI, T2, T3 refer to the three waves of the study. GPA = grade point average.

$* * p<.01$. ***p $<.001$

middle-school year. Students who were absent on the day of testing were tested on a later day within a week (group or individual testing). Immigrant students could choose the language in which they preferred to respond to the questionnaires. Less than $5 \%$ of the students opted for an Albanian or Russian version. These versions were prepared with the help of four bilingual speakers using back-translation. In addition, the Greek language teacher in each classroom was asked to evaluate students' conduct and school engagement, and students' grades were obtained from school records.

\section{Results}

\section{Overview}

First, we present the descriptives and intercorrelations of the main variables, and information about missing data. Because all three hypotheses were tested using cross-lagged regression models, we highlight key requirements for the interpretation of cross-lagged analyses and describe how we dealt with them in the present study. Third, we present the results of the tests of Hypothesis 1-3 and the exploratory analyses.

\section{Descriptives and Intercorrelations of the Main Variables}

The means and initial correlations of the main variables are shown in Table 1 for descriptive purposes. Note that the means for the preference scores are not informative because the students were asked to provide three positive and three negative nominations such that their difference was close to zero for each nominator and therefore also overall for the nominees.

\section{Missing Data}

Table 1 indicates that missing data except for those due to attrition ( $8 \%$ in Wave 2, additional $8 \%$ in Wave 3, see sample description) were not frequent. Dropout effects were studied by comparing the dropouts over the course of the study with the remaining participants using $t$-tests. In addition to the main variables listed in Table 1, we also studied dropout effects for immigrant status and gender. To control for alpha inflation due to the many tests, we used $p=.01$ as the critical value for a significant dropout effect. Four significant effects were found. Dropouts were judged as less engaged in school, $t(1,076)=12.37, p<.001, d=0.75$, had lower grades, $t(1,113)=7.85, p<.001, d=0.47$, were more frequent immigrants, $t(1,116)=4.17, p<.001, d=0.25$, and were less preferred by their classmates, $t(1,044)=2.84, p<.01, d=0.18$. Whereas the dropout effects for peer preferences and adaptation were fully controlled by using Full Information Maximum Likelihood (FIML) estimation in the cross-lagged analyses, immigrant status was not included in these analyses. Therefore, we ran all models also with immigrant status as an auxiliary variable. The results were virtually identical such that the missing at random assumption for the cross-lagged analyses was supported.

In addition to these descriptive data at the individual level, we report descriptive data for the classroom level that is important for the sociometric nominations. In Wave 1, classroom size varied between 11 and 27 students, $M=19.61, S D=3.42$. The percentage of students participating in the nominations varied between $71 \%$ and $100 \%, M=92 \%$, with a slightly higher participation rate for Greeks (94\%) compared to immigrants (91\%). In the following waves, the differences in classroom sizes were somewhat smaller, varying from 13 to 26 , and the participation rates decreased slightly for immigrants to $86 \%$ and to $88 \%$ for Greeks. Participation rates were above $70 \%$ for all classrooms and waves for both Greeks and immigrants.

\section{Cross-Lagged Regression Analyses}

The longitudinal design of our study made it possible to disentangle the effects of preferences by peers on adaptation and well-being from the reverse effects of adaptation and well-being on preferences with cross-lagged regression models (see e.g., Little, 2013). The effect of $\mathrm{X}$ on $\mathrm{Y}$ is estimated by the direct path from $\mathrm{X}$ in Wave $\mathrm{T}$ to $\mathrm{Y}$ in Wave $\mathrm{T}+1$, controlling for the stability of $\mathrm{Y}$ (thus controlling for the indirect path from $\mathrm{X}$ to $\mathrm{Y}$ at $\mathrm{T}+1$ through $\mathrm{Y}$ at $\mathrm{T}$ ), and vice versa for the effect of $\mathrm{Y}$ on $\mathrm{X}$. The residuals for $\mathrm{X}$ and $\mathrm{Y}$ in Wave 2 and later are allowed to correlate; they capture effects of unmeasured variables on $\mathrm{X}$ and $\mathrm{Y}$ that are shared by $\mathrm{X}$ and $\mathrm{Y}$. A significant cross-path from $\mathrm{X}$ to $\mathrm{Y}$ indicates that $\mathrm{X}$ has an effect on the residualized change in $\mathrm{Y}$ between the current wave and the next wave. The effects of $\mathrm{X}$ on $\mathrm{Y}$ and of $\mathrm{Y}$ on $\mathrm{X}$ can be compared in terms of their effect size and significance. Thereby cross-lagged 
Table 2. Relations between preference by peers and adaptation and well-being by immigrant status.

\begin{tabular}{|c|c|c|c|c|c|}
\hline \multirow{2}{*}{$\begin{array}{l}\text { Adaptation and } \\
\text { well-being } Y\end{array}$} & \multirow[b]{2}{*}{$r_{\mathrm{TI}}$} & \multicolumn{2}{|c|}{ Unstandardized solution } & \multicolumn{2}{|c|}{ Standardized solution } \\
\hline & & $b \mathrm{PR}_{\mathrm{T}} \rightarrow \mathrm{Y}_{\mathrm{T}+1}$ & $b \mathrm{Y}_{\mathrm{T}} \rightarrow \mathrm{PR}_{\mathrm{T}+1}$ & $\beta \mathrm{PR}_{\mathrm{T}} \rightarrow \mathrm{Y}_{\mathrm{T}+1}$ & $\beta \mathrm{Y}_{\mathrm{T}} \rightarrow \mathrm{PR}_{\mathrm{T}+1}$ \\
\hline \multicolumn{6}{|l|}{ Preference by all peers } \\
\hline Self-esteem & $.117[-.071, .305]$ & $.080[-.047, .207]$ & $-.005[-.031, .021]$ & $.024[-.009, .057]$ & $-.017[-.094, .060]$ \\
\hline Depression & $-.052[-.113, .009]$ & $-.097[-.184,-.010]$ & $.009[-.003, .021]$ & $-.035[-.066,-.004]$ & $.023[-.010, .056]$ \\
\hline Conduct & $.250[.202, .298]$ & $.424[.28 \mathrm{I}, .567]$ & $.016[.008, .024]$ & $.134[.085, .183]$ & $.06 \mathrm{I}[.028, .094]$ \\
\hline School engagement & $.301[.250, .352]$ & $.355[.204, .506]$ & $.016[.009, .023]$ & $.077[.044, .110]$ & $.076[.043, .109]$ \\
\hline School achievement & $.361[.320, .402]$ & $-.148[-.359, .063]$ & $.009[.007, .011]$ & $-.010[-.025, .005]$ & $.132[.096, .168]$ \\
\hline \multicolumn{6}{|l|}{ Preference by Greeks } \\
\hline Self-esteem & $.067[.003, .131]$ & $.088[.009, .167]$ & $-.001[-.024, .026]$ & $.043[.007, .079]$ & $-.003[-.057, .05 \mathrm{I}]$ \\
\hline Depression & $-.042[-.101, .017]$ & $-.061[-.125, .003]$ & $-.011[-.029, .007]$ & $-.033[-.068, .002]$ & $-.021[-.059, .017]$ \\
\hline Conduct & $.212[.169, .255]$ & $.167[.083, .251]$ & $.034[.018, .050]$ & $.082[.036, .128]$ & $.092[.053, .131]$ \\
\hline School engagement & $.265[.211, .319]$ & $.264[.155, .373]$ & $.026[.014, .038]$ & $.088[.053, .123]$ & $.092[.053, .131]$ \\
\hline School achievement & $.298[.242, .354]$ & $.058[-.090, .206]$ & $.016[.011, .021]$ & $.007[-.009, .023]$ & $.163[.119, .207]$ \\
\hline \multicolumn{6}{|c|}{ Preference by immigrants } \\
\hline Self-esteem & $.107[-.026, .240]$ & $.020[-.082, .122]$ & $-.004[-.020, .012]$ & $.007[-.026, .040]$ & $-.013[-.059, .033]$ \\
\hline Depression & $-.053[-.112, .006]$ & $-.073[-.154, .008]$ & $.010[-.006, .026]$ & $-.029[-.060, .002]$ & $.025[-.014, .064]$ \\
\hline Conduct & $.192[.141, .243]$ & $.362[.250, .474]$ & $.020[.010, .030]$ & $.123[.084, .162]$ & $.071[.036, .106]$ \\
\hline School engagement & $.200[.149, .251]$ & $.203[.086, .320]$ & $.019[.011, .027]$ & $.048[.018, .078]$ & $.083[.045, .121]$ \\
\hline School achievement & $.264[.216, .312]$ & $-.159[-.381, .063]$ & $.010[.007, .013]$ & $-.012[-.028, .004]$ & $.125[.094, .156]$ \\
\hline
\end{tabular}

Note. $N=1$ I I8. Reported are initial Pearson correlations $r_{\mathrm{Tl}}$, unstandardized regression coefficients $b$, and standardized cross-lagged regression coefficients $\beta$ for effects of preferences by peers PR at time T on outcomes $Y$ at time $\mathrm{T}+\mathrm{I}$ and vice versa in cross-lagged regression models. Confidence intervals are $90 \% \mathrm{Cls}$ (one-tailed tests). Data are controlled for the clustering of students in classrooms.

models can disentangle the two directions of the effects underlying the correlation of $\mathrm{X}$ and $\mathrm{Y}$.

All models were analyzed using MPlus 7.0 (Muthén \& Muthén, 1998-2012). Selective attrition in the variables included in the cross-lagged models was controlled by using FIML estimation that corrects for the influence of missing values both within waves and across waves, and adding immigrant status as an additional auxiliary variable (see section on missing data). Because the data had a nested structure (students nested in classrooms) and the betweenclassroom variance was significant $(p<.001)$ for the teacher-based variables conduct, school engagement, and GPA, we controlled for cluster effects using the TYPE $=$ COMPLEX option of MPlus 7 in all cases. Simpler ordinary regression analyses that ignored the nesting of students in classrooms produced virtually identical results. All analyses were based on robust standard errors (MLR estimator) which is a requirement in MPlus for the TYPE $=$ COMPLEX option. Therefore, relative model fit could not be evaluated with the conventional $\chi^{2}$ difference test. Following recommendations by Kline (2016), we used the CFI difference as a measure of relative model fit. Differences up to .01 can be tolerated.

Because the measures of adaptation and well-being showed good internal consistencies (see "Method" section), we controlled for unreliability by using latent variables with two item parcels as indicators. These parcels correlated above .68 for each measure of adaptation and well-being for each wave of the study. Analyses of measurement equivalence showed that we could use the same parcel loadings on the latent variable across time. We allowed the individual indicators to correlate across time to allow for item-specific stability that is not captured by the stability of the latent variables.

A total of 5 (adaptation, well-being) $\times 3$ (all, Greek, immigrant nominees) $=15$ cross-lagged analyses were run, first with unconstrained paths. The fit of these models was good in each case, with RMSEA < .05 and CFI > .95 (Hu \& Bentler, 1999). Subsequently, we tested whether the lagged cross-paths were stationary (not different across time). Stationarity is a reasonable hypothesis in the present case because of the relatively short retest interval of 1 year and the fact that the large majority of the students remained in the same peer group from one year to the next (stable social environment). If stationarity can be assumed, the two corresponding lagged cross-paths can be averaged which increases their reliability. We compared the fit of each model with the fit of a more restricted model where the two lagged cross-paths between $\mathrm{T} 1$ and $\mathrm{T} 2$ were set equal to the corresponding paths from T2 to T3. Because $\Delta$ CFI was below .01 in all 15 cases, we used the constrained models as the final models.

The results of the 15 analyses are reported in Table 2 in terms of the two lagged cross-paths from preference to adaptation and well-being, and vice versa. Although the estimated initial correlations were highly similar to those reported in Table 1, we also report them in order to facilitate the interpretation of the results. The effect sizes of the paths were reported in terms of both unstandardized and standardized regression coefficients such that the latter can be directly compared between different measures. Due to differences in variance, the standardized effect sizes can slightly differ between two paths that were set equal; we report in Table 2 the mean of these two effect sizes and the mean of their CIs. It should be noted that cross-lagged regressions are first-order regressions controlled for the often high autoregressions of the variables. Therefore, their effect sizes may seem relatively small compared to effect sizes for zero-order regressions but this comparison is not recommended because their size is limited by the autoregressions (see e.g., Adachi \& Willoughby, 2015).

Because the sign of the initial correlations and the cross-lagged effects was expected a priori to be positive between preference and both adaptation and well-being (and negative for depression) for both effects from and to preference, one-tailed tests were used and $90 \%$ CIs are reported. Significant effects are indicated by the CI not including zero. The results listed in Table 2 showed that all significant effects were positive (and negative for depression), validating the choice of one-tailed tests. 

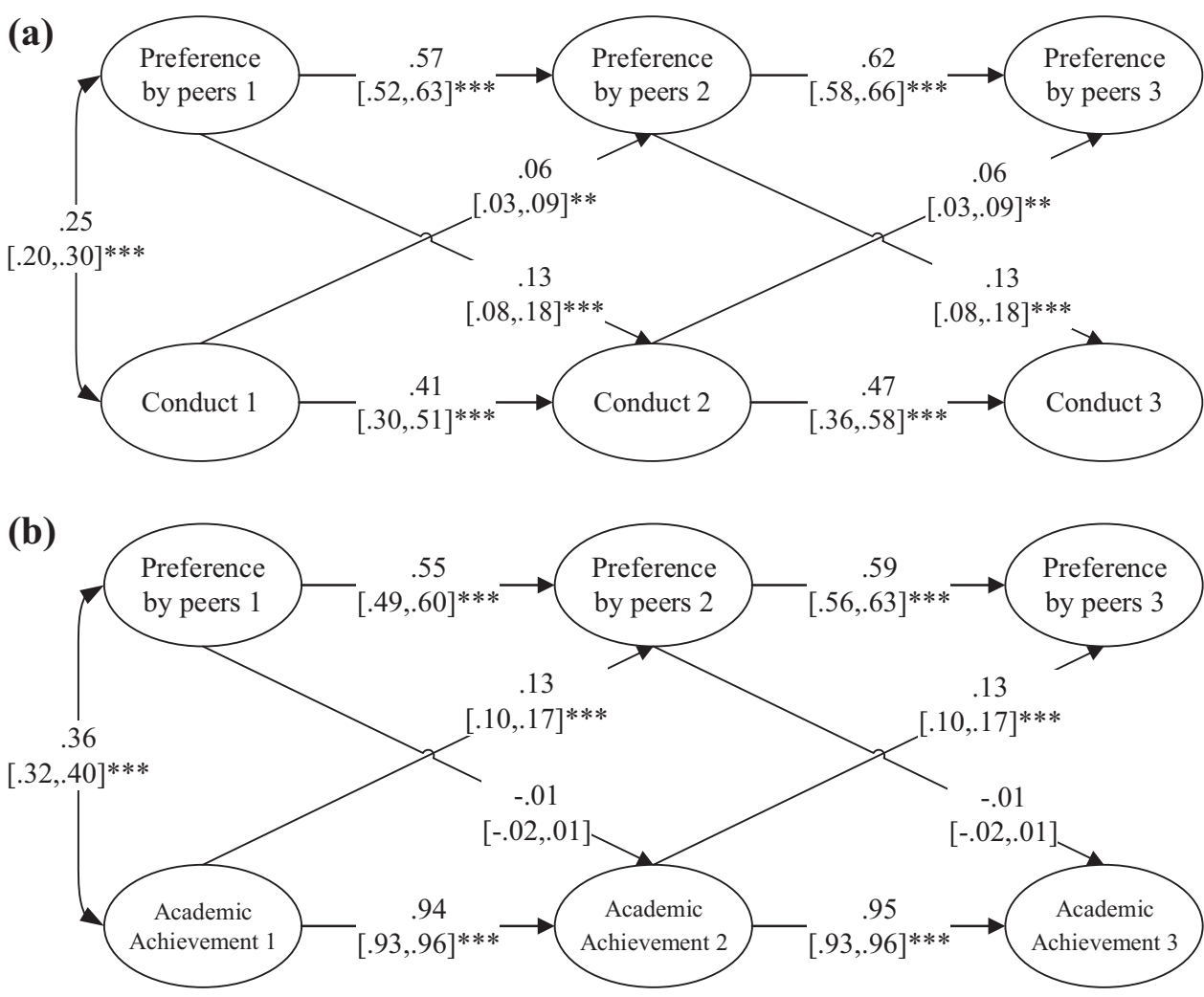

Figure I. Two types of relations between preference by peers and adaptation (standardized solution of cross-lagged regression models). Confidence intervals and significances refer to one-tailed tests. $*_{p} p<01 . * * * p<.001$. (a) Bidirectional effects between preference by peers and conduct. (b) Bidirectional effects between preference by peers and academic achievement.

\section{Links Between Peer Preferences and Youth's Adaptation and Well-Being}

The first hypothesis, stating that the effects between being preferred by peers and conduct would be bidirectional, was confirmed for the total sample. Both the effect of peer preference on later conduct and the effect of conduct on later peer preference were significant, with effect sizes of $\beta=.134$ and $\beta=.061$, see Table 2 and Figure 1(a)). Thus, Hypothesis 1 was fully confirmed.

The second hypothesis, stating that peer preferences would decrease later depression and increase later self-esteem and school engagement, was confirmed for depression and school engagement but not for self-esteem (see Table 2).

The third hypothesis, stating that academic achievement would increase later peer preferences but not vice versa, was also confirmed (see Table 2 and Figure 1(b)).

Regarding the remaining effects in the opposite direction, school engagement increased over time peer preferences (in line with academic achievement) whereas both self-esteem and depression did not show any significant effect (see Table 2).

\section{Moderation by Immigrant Status}

The fourth hypothesis stating that Greek students' preferences would have stronger effects on school adjustment and well-being than immigrant students' preferences tended to be supported for both measures of well-being. The differences in effect size for both self-esteem and depression were in line with Hypothesis 4. Greeks' preferences significantly predicted students' self-esteem (and marginally students' depression, $p=.058$ ) whereas the effects of immigrants' preferences on self-esteem and depression were nonsignificant in both cases (marginally for students' depression, $p=.070)$. It should be noted that the preferences by Greeks were less reliably estimated than the preferences by immigrants due to the smaller number of Greek nominators such that significant effects were less likely for them. The difference between Greeks' and immigrants' preferences could not be tested for significance because the bivariate cross-lagged models including both dependent variables were not estimable due to too many parameters. In contrast, Hypothesis 4 was not supported for school adjustment (conduct, engagement and achievement). As Table 2 shows, the predictions tended to be sometimes stronger for Greeks' preferences and sometimes for immigrants' preferences.

\section{Further Exploratory Analyses}

To examine whether the cross-lagged effects for Greek versus immigrant nominees were different, we ran multigroup analyses that compared the cross-lagged effects in the 15 analyses reported in Table 2 between Greek and immigrant students. The CFI differences between the two groups of nominees were far from being meaningful in each case $(\Delta \mathrm{CFI}<.001)$. Thus, the cross-lagged effects were not different between Greek and immigrant nominees. 


\section{Discussion}

The focus of the present study was two-fold. We examined the longitudinal interplay between peer preferences and, youth's school adjustment and psychological well-being, and, whether immigrant status differentiated this link. We tested for bidirectional influences between these variables using complex dynamic models.

In the total sample, we found that positive and negative experiences in the peer context, in the form of peer preferences, influenced over time a number of indices of youth's school adjustment and well-being. In the opposite direction, a number of indices of school adjustment and well-being had an effect over time on whether youth are liked by their classmates, revealing the role of adaptive history for youth's current adaptation with respect to peer relations. We also found that only peer preferences by Greek peers predicted their classmates' well-being from one year to the next, and that peer preferences by both Greek and immigrant students equally affected longitudinally their classmates' school adjustment.

\section{Links Between Peer Preferences and Youth's Adaptation}

The first question addressed was whether and how peer preferences and youth's school adjustment and well-being are longitudinally linked. It addresses the total sample, including both Greek and immigrant students.

As expected, the results reveal bidirectional effects over time between peer preferences and conduct. Higher peer preferences led in both time windows to better conduct, but better conduct also led to higher peer preferences. Thus, the results reveal a transactional pattern (Sameroff, 1975) between peer preferences and youth's conduct, whereby peer preferences influence over time youth's conduct, which in turn further contributes to the construction of their social context, and vice versa. Our findings are in line with other studies from North America with younger children showing that peer preferences and aggression influence each other reciprocally over time (Lansford et al., 2010). They make a novel contribution to the literature in demonstrating that the nature of these links is similarly dynamic and complex in early adolescence and in a different cultural context.

The results need to be interpreted in the Greek school context. Middle-school students in Greece stay together in the same classroom throughout the day during the 3 years of middle school. Teachers come to their classroom to teach different subjects. In Wave 1 of the study, participants had just transitioned from primary to middle school. Although they are placed with new classmates in classrooms which are newly formed, they come into middle school with a peer relationship history. Some have already experienced peer rejection in the past which is considered to be a major adversity in children's lives (e.g., Lansford et al., 2010). As a result, these youths may arrive in middle school with externalizing problems.

However, the effect from conduct to peer preferences was only half the size of the effect from peer preferences to conduct. Since youth have recently been placed in these classrooms with new classmates, it may be argued that current peer rejection compounds the relationship baggage that they bring to middle school from primary school. In accordance with the SIP model (Dodge \& Crick, 1990), one could advance the hypothesis that their relationship history (from early negative peer preferences to conduct problems) possibly contributes to a negative interpretation of current ambiguous events which leads to a new cycle of peer rejection and further increases in aggression.

As expected, negative peer preferences led longitudinally to increases in depressive symptoms. The other direction of effects from depressive symptoms to peer preferences was not significant. Cognitive biases in encoding and interpreting social cues may be implicated in the link between negative peer preferences and depressive symptoms (Crick \& Dodge, 1994). Youth who interpret ambiguous social stimuli as due to perceived self-deficits (NolenHoeksema et al., 1992), or who interpret stressful life experiences, such as negative peer preferences, as due to internal, global, and stable causes, may be more at risk to develop depressive symptoms and withdrawal (Prinstein et al., 2018).

As expected, peer preferences did not predict over time changes in youth's academic achievement. This finding is most probably due to the very high stability of academic achievement over the three years of middle school. However, peer preferences longitudinally predicted changes in school engagement. Being preferred by peers led a year later to increases in school engagement.

Peer relations may contribute to youth's academic motivation by communicating educational goals and expectations for performance, by providing access to resources and information, as well as by making youth feel emotional security and social belonging. These mechanisms facilitate the adoption of goals and interests valued by others (Wentzel, 2009).

Finally, both higher academic achievement and school engagement predicted a year later increases in peer preferences. This finding could reflect early adolescents' expectations that their classmates perform well academically at school (Wentzel, 2009).

It should be noted that all students' school adjustment, but not their well-being, showed longitudinal effects on peer preferences. A post-hoc explanation may be that all three indices of school adjustment refer to observable behaviors or achievements, which may be valued, whereas psychological well-being may not be easily perceived by peers. Furthermore, since peer preference scores are based on a peer sociometric test, it is possible that the aggressive conduct of certain students may affect negatively their classmates who are evaluating their likeability.

\section{Disaggregating the Link Between Peer Preference and Adaptation by Immigrant Status}

Who drives the effects of peer preferences on immigrant youth's adaptation? Based on the higher status of Greeks in society at large (Pavlopoulos \& Motti-Stefanidi, 2017), and, relatedly, their higher social status in their classrooms (Asendorpf \& Motti-Stefanidi, 2017) we expected that preference by Greek students has stronger effects on the school adjustment and well-being of their classmates. Our hypotheses were partially confirmed.

As expected, being preferred by Greek peers, but not being preferred by immigrant peers, tended to predict one year later higher self-esteem and fewer depressive symptoms among all classmates. As Verkuyten and colleagues (1996) have shown, in multiethnic societies, ethnic majority members are considered to have a higher standing in society compared to ethnic minority members. Furthermore, Greek students are also more liked and less disliked by their classmates, a finding that was attributed to their higher societal status (Asendorpf \& Motti-Stefanidi, 2017). Groups who have a higher social status in their classrooms have been shown to be more powerful agents of socialization than low status groups 
(Ellis \& Zarbatany, 2007). Based on these arguments, the hypothesis can be formulated that Greeks' higher societal status influences over time their likeability among their classmates (Asendorpf \& Motti-Stefanidi, 2017), which in turn confers them the power to influence their classmates' well-being (see Laursen, 2018).

The results of the present study regarding the link between peer preferences and self-esteem are only partially consistent with the findings by Reitz et al. (2016) which were based on data from an earlier AStRA cohort. First, in the present study, unexpectedly, we did not find a longitudinal link between peer preferences and selfesteem. Second, Reitz et al. (2016) reported that preference by the in-group, but not by the out-group, increased youth's later selfesteem. In contrast, in this study, we did not find an in-group or an outgroup effect on self-esteem. Instead we found that being preferred by Greek students predicted over time high self-esteem for both Greek and immigrant students. The higher ethnic diversity and higher proportion of immigrants in the classrooms of the present cohort (see Motti-Stefanidi \& Asendorpf, 2017) may partly explain the inconsistency with the Reitz et al. (2016) findings. This hypothesis needs to be further explored in the future.

Unexpectedly, peer preferences by both Greek and immigrant students equally predicted their classmates' teacher-rated conduct and school engagement. A post-hoc explanation of this difference in the effect of peer preferences on school adjustment, which is teacher-rated, versus psychological well-being, which is selfreported, may be that teacher ratings of students' conduct and school engagement are more objective and independent of whether these students are preferred by Greek or immigrant students. In contrast, students' self-reported well-being seems to be directly affected by who nominates them possibly because there is a social power differential in the classrooms between Greek and immigrant students.

Regarding the opposite direction of influence, both Greek and immigrant youth's school adjustment consistently influenced changes in peer preferences. Being a better student and more motivated, and exhibiting better conduct led over time to being more preferred by one's classmates. All three are signs of positive adaptation with respect to developmental tasks for both Greeks and immigrants, alike (Masten, 2014). These markers of positive adaptation seem to be recognized as such and valued by all students (Wentzel, 2009). In the case of immigrant youth, doing well with respect to these tasks requires that they have acquired sufficient competence in the academic language of the host country to do well enough in school and that they have accepted the rules of the school and host society. Thus, for immigrant youth they are also a sign that they are well adapted with respect to acculturative tasks (Motti-Stefanidi et al., 2012; Suarez-Orozco et al., 2018). In this line, in another study based on the same data, we found that immigrant youth's higher acculturation into the Greek culture predicted increases in acceptance by Greek peers, which in turn decreased perceptions of discrimination (Motti-Stefanidi et al., 2018).

\section{Strengths and Limitations}

A strength of this study is that it allowed conceptually and methodologically to test the longitudinal links between peer likeability and a wide range of school adjustment and well-being indices in a sample of youth living in another culture than the U.S. where most peer relations studies have been conducted. It also allowed to disaggregate the data by immigrant status and to examine how it affects the links between peer likeability and adaptation, which has significant implications for intergroup relations.

Conceptually, the study is framed within an overarching developmental resilience model, which includes social psychological and acculturation perspectives to account for the diversity in immigrant youth adaptation (Motti-Stefanidi et al., 2012; Suárez-Orozco et al., 2018). The bidirectionality developmental principle guided the research questions and the interpretation of the results both for the total sample, including immigrant and nonimmigrant youth, and for each group separately.

In terms of methodology, the present study has a number of strengths: systematic sampling of schools in neighborhoods with many immigrants; large sample of both students and classrooms; relating peer judgments to teacher- and self-judgments which avoids problems of shared method variance; following a longitudinal sample from the first year in middle school to the third year which allows for studying the building of peer relationships in a new peer group; cross-lagged analyses that can disentangle directions of effects on inter-individual differences in change.

Limitations are the specific culture and involved ethnicities and the specific historical condition of that culture, which during data collection was in the middle of a severe economic crisis. However, it should be noted that Greece is a European country. Many European countries hold prejudice against, and have negative attitudes toward the presence of, immigrants (OECD, 2015), with significant consequences for immigrant youth's adaptation and well-being (Motti-Stefanidi et al., 2012).

Another limitation is that in some classrooms the number of Greek nominators was small such that the preference by Greeks could not be assessed in these classrooms with high reliability. We did not exclude those classrooms because this might induce a systematic bias in the results (thus, we faced a reliability-validity trade-off). Instead we accepted the limited reliability of the preference scores in these cases.

A final limitation was that differences between the effects of preferences by Greek versus immigrant students could not be tested for significance such that we had to rely on differences in magnitude only.

\section{Conclusions}

Adolescents live in peer-rich social worlds characterized by great diversity. The quality of their relations with peers is consequential for their development and adaptation. The results of this study add to the extant peer relations literature by focusing on the link between peer likeability and adaptation in youth living in poor areas, with many migrants, of a large city of a Southern European country. In line with other studies mostly conducted in the U.S., they reveal that being liked by one's peers is for all youth a contributor to, as well as a manifestation of, positive school adjustment and well-being. However, in this multicultural world, youth often need to learn to navigate between inter-ethnic and intra-ethnic peers. The results suggest that being liked particularly by Greeks is consequential for their classmates' well-being.

The results may inform public policy and interventions in the school context. Policies that promote respect and tolerance in the relationships between youth and their peers, and interventions that do not leave peer relationships to chance but structure classrooms and plan activities in ways that offer the opportunity for intergroup contact, may lead to more acceptance of diversity and less fear of 
the other, which may affect youth's future development and adaptation in substantial ways.

\section{Authors' Note}

The present study is part of the Athena Studies of Resilient Adaptation (AStRA), a collaborative project focusing on the quality of adaptation of immigrant youth living in Greece.

\section{Author Contributions}

The first and the last authors equally contributed to this article.

\section{Note}

Frosso Motti-Stefanidi delivered an invited address at the 2018 biennial meetings in Gold Coast, Australia. This invited paper was based on that invited talk. Invited articles are reviewed, but the review process differs from that of unsolicited articles in that the goal is to improve the impact and appeal of the submission.

\section{Funding}

The author(s) disclosed receipt of the following financial support for the research, authorship, and/or publication of this article: This project was supported by an Excellence grant to Frosso MottiStefanidi, co-funded by the European Social Fund and Greek National Resources (ESPA-Excellence II).

\section{ORCID iD}

Frosso Motti-Stefanidi (D) https://orcid.org/0000-0003-4290-4895

\section{References}

Adachi, P., \& Willoughby, T. (2015). Interpreting effect sizes when controlling for stability effects in longitudinal autoregressive models: Implications for psychological science. European Journal of Developmental Psychology, 12, 116-128. https://doi.org/10.1080/ 17405629.2014.963549

Asendorpf, J. P., \& Motti-Stefanidi, F. (2017). A longitudinal study of immigrants' peer acceptance and rejection: Immigrant status, immigrant composition of the classroom, and acculturation. Cultural Diversity and Ethnic Minority Psychology, 23, 486-498. https:// doi.org/10.1037/cdp0000155

Bronfenbrenner, U., \& Morris, P. A. (2006). The bioecological model of human development. In R. M. Lerner (Ed.), Handbook of child psychology. Vol. 1: Theoretical models of human development (6th ed., pp. 793-828). Wiley.

Cicchetti, D., \& Rogosh, F. A. (2002). A developmental psychopathology perspective on adolescence. Journal of Consulting and Clinical Psychology, 70, 6-20. https://doi.org/10.1037/0022-006X.70.1.6

Cillessen, A. H. N., \& Marks, P. E. L. (2011). Conceptualizing and measuring popularity. In A. H. N. Cillessen, D. Schwartz, \& L. Mayeux (Eds.), Popularity in the peer system (pp. 25-56). Guilford Press.

Crick, N. R., \& Dodge, K. A. (1994). A review and reformulation of social information-processing mechanisms in children's social adjustment. Psychological Bulletin, 115, 74-101.

Dodge, K. A., \& Crick, N. R. (1990). Social information-processing bases of aggressive behavior in children. Personality and Social Psychology Bulletin, 16, 8-22. https://doi.org/10.1177/0146167290161002

Ellis, W. E., \& Zarbatany, L. (2007). Peer group status as a moderator of group influence on children's deviant, aggressive, and prosocial behavior. Child Development, 78, 1240-1254. https://doi.org/10. 1111/j.1467-8624.2007.01063.x
Finan, L. J., Ohannessian, C. M., \& Gordon, M. S. (2018). Trajectories of depressive symptoms from adolescence to emerging adulthood: The influence of parents, peers, and siblings. Developmental Psychology, 54, 1555-1567. https://doi.org/10.1037/dev0000543

Hu, L. T., \& Bentler, P. M. (1999). Cutoff criteria for fit indexes in covariance structure analysis: Conventional criteria versus new alternatives. Structural Equation Modeling, 6, 1-55. https://doi. org/10.1080/10705519909540118

Jackson, M. F., Barth, J. M., Powell, N., \& Lochman, J. E. (2006). Classroom contextual effects of race on children's peer nominations. Child Development, 77, 1325-1337. https://doi.org/10.1111/ j.1467-8624.2006.00937

Jiang, X. L., \& Cillessen, A. H. N. (2005). Stability of continuous measures of sociometric status: A meta-analysis. Developmental Review, 25, 1-25. https://doi.org/10.1016/j.dr.2004.08.008

Kennedy, E., Spence, S. H., \& Hensley, R. (1989). An examination of the relationship between childhood depression and social competence amongst primary school children. Journal of Child Psychology and Psychiatry, 30, 561-573. https://doi.org/10.1111/j.1469-7610.1989. tb00268.x

Kline, R. B. (2016). Principles and practice of structural equation modeling (4th ed.). Guilford Press.

Lansford, J. E., Malone, P. S., Dodge, K. A., Pettit, G. S., \& Bates, J. E. (2010). Developmental cascades of peer rejection, social information processing biases, and aggression during middle childhood. Development and Psychopathology, 22, 593-602. https://doi.org/ 10.1017/S0954579410000301

Laursen, B. (2018). Peer influence. In W. M. Bukowski, B. Laursen, \& K. H. Rubin (Eds.), Handbook of peer interactions, relationships, and groups (Vol. 2, pp. 447-469). Guilford Press.

Leary, M. R., \& Baumeister, R. F. (2000). The nature and function of self-esteem: Sociometer theory. In M. P. Zanna (Ed.), Advances in experimental social psychology (Vol. 32, pp. 1-62). San Diego, CA: Academic Press. https://doi.org/10.1016/S0065-2601(00)80003-9.

Little, T. D. (2013). Longitudinal structural equation modeling. Guilford Press.

Marks, A. K., Ejesi, K., McCullough, M. B., \& García Coll, C. (2015). Developmental implications of discrimination. In R. M. Lerner (Ed.), Handbook of child psychology and developmental science: Vol. 3. Socioemotional processes (7th ed., pp. 1-42). Wiley \& Sons.

Masten, A. S. (2014). Ordinary magic: Resilience in development. Guilford Press.

Masten, A. S., \& Kalstabakken, A. W. (2018). Developmental perspectives on psychopathology in children and adolescents. In J. M. Butcher (Ed.), APA handbook of psychoopathology. Vol. 2. Child and adolescent psychopathology (pp. 15-36). American Psychological Association.

Motti-Stefanidi, F. (2018). Resilience among immigrant youth: The role of culture, development and acculturation. Developmental Review, 50, 99-109. https://doi.org/10.1016/j.dr.2018.04.002

Motti-Stefanidi, F., Asendorpf, J. B., \& Masten, A. S. (2012). The adaptation and well-being of adolescent immigrants in Greek schools: A multilevel, longitudinal study of risks and resources. Development and Psychopathology, 24, 451-473. https://doi.org/ 10.1017/S0954579412000090

Motti-Stefanidi, F., Berry, J. W., Chryssochoou, X., Sam, D. L., \& Phinney, J. S. (2012). Positive immigrant youth adaptation in context: Developmental, acculturation, and social-psychological perspectives. In A. S. Masten, K. Liebkind, \& D. J. Hernandez (Eds.), Realizing the potential of immigrant youth (pp. 117-158). Cambridge University Press. 
Motti-Stefanidi, F., \& Masten, A. S. (2017). A resilience perspective on immigrant youth adaptation and development. In N. J. Cabrera \& B. Leyendecker (Eds.), Handbook on positive development of minority children and youth (pp. 19-34). Springer.

Motti-Stefanidi, F., Pavlopoulos, V., \& Asendorpf, J. B. (2018). Immigrant youth acculturation and perceived discrimination: Longitudinal mediation by immigrant peers' acceptance/rejection. Journal of Applied Developmental Psychology, 59, 36-45. https://doi.org/10. 1016/j.appdev.2018.03.001

Muthén, L. K., \& Muthén, B. O. (1998-2012). Mplus version 7. Author.

Nolen-Hoeksema, S., Girgus, J. S., \& Seligman, M. E. (1992). Predictors and consequences of childhood depressive symptoms: A 5-year longitudinal study. Journal of Abnormal Psychology, 101, 405-422. https://doi.org/10.1037/0021-843X.101.3.405

Organization for Economic Cooperation and Development. (2016, September 9). Governments must address anti-immigration backlash. Author.

Organization for Economic Cooperation and Development \& European Union. (2015). Indicators of immigrant integration 2015: Settling in. OECD Publishing. https://doi.org/10.1787/9789264234024-en

Pavlopoulos, V., \& Motti-Stefanidi, F. (2017). Mutual intercultural relations in Greece. In J. Berry (Ed.), Mutual intercultural relations (pp. 187-209). Cambridge University Press.

Prinstein, M. J., Rancourt, D., Adelman, C. B., Ahlich, E., Smith, J., \& Guerry, J. D. (2018). Peer status and psychopathology. In W. M. Bukowski, B. Laursen, \& K. H. Rubin (Eds.), Handbook of peer interactions, relationships, and groups (Vol. 2, pp. 617-636). Guilford Press.

Reitz, A. K., Motti-Stefanidi, F., \& Asendorpf, J. B. (2016). Me, us, and them: Testing sociometer theory in a socially diverse real-life context. Journal of Personality and Social Psychology, 110, 908-920. https://doi.org/10.1037/pspp0000073

Robins, R. W., Hendin, H. M., \& Trzesniewski, K. H. (2001). Measuring global self-esteem: Construct validation of a single-item measure and the Rosenberg self-esteem scale. Personality and Social Psychology Bulletin, 27, 151-161. https://doi.org/10.1177/ 0146167201272002

Rosenberg, M. (1965). Society and the adolescent self-image. Princeton University Press.

Rubin, K. H., Bukowski, W. M., \& Bowker, J. C. (2015). Children in peer groups. In R. M. Lerner, M. H. Bornstein, \& T. Leventhal (Eds.), Handbook of child psychology and developmental science: Vol. 4. Ecological settings and processes (7th ed., pp. 175-222). John Wiley.

Rubin, K. H., Coplan, R. J., \& Bowker, J. C. (2009). Social withdrawal in childhood. Annual Review of Psychology, 60, 141-171. https:// doi.org/10.1146/annurev.psych.60.110707.163642
Ryan, A. M., \& Shin, H. (2018). Peers, academics and teachers. In W. M. Bukowski, B. Laursen, \& K. H. Rubin (Eds.), Handbook of peer interactions, relationships, and groups (Vol. 2, pp. 637-656). Guilford Press.

Salokangas, R. K., Poutanen, O., \& Stengård, E. (1995). Screening for depression in primary care: Development and validation of the depression scale, a screening instrument for depression. Acta Psychiatrica Scandinavica, 92, 10-16. https://doi.org/10.1111/j.16000447.1995.tb09536.x

Sameroff, A. (1975). Transactional models in early social relations. Human Development, 18, 65-79. https://doi.org/10.1159/ 000271476

Sentse, M., Kretschmer, T., \& Salmivalli, C. (2015). The longitudinal interplay between bullying, victimization, and social status: Age-related and gender differences. Social Development, 24, 659-677. https://doi.org/10.1111/sode.12115

Sroufe, L. A., Egeland, B., Carlson, E. A., \& Collins, W. A. (2005). The development of the person: The Minnesota study of risk and adaptation from birth to adulthood. Guilford.

Suárez-Orozco, C., Motti-Stefanidi, F., Marks, A., \& Katsiaficas, D. (2018). An integrative risk and resilience model for understanding the adaptation of immigrant-origin children and youth. American Psychologist, 73, 781-796. https://doi.org/10.1037/ amp0000265

Triandafyllidou, A. (2014). Migration in Greece: Recent developments in 2014. Athens: Hellenic Foundation for European \& Foreign Policy. http://www.eliamep.gr/wp-content/uploads/2014/10/Migra tion-in-Greece-Recent-Developments-2014_2.pdf

Tseng, W. L., Banny, A. M., Kawabata, Y., Crick, N. R., \& Gau, S. S. F. (2013). A cross-lagged structural equation model of relational aggression, physical aggression, and peer status in a Chinese culture. Aggressive Behavior, 39, 301-315. https://doi.org/10.1002/ab. 21480

Verkuyten, M., Hagendoorn, L., \& Masson, K. (1996). The ethnic hierarchy among majority and minority youth in the Netherlands. Journal of Applied Social Psychology, 26, 1104-1118. https://doi. org/10.1111/j.1559-1816.1996.tb01127.x

Véronneau, M. H., Vitaro, F., Brendgen, M., Dishion, T. J., \& Tremblay, R. E. (2010). Transactional analysis of the reciprocal links between peer experiences and academic achievement from middle childhood to early adolescence. Developmental Psychology, 46, 773. https://doi.org/10.1037/a0019816

Wentzel, K. R. (2009). Peers and academic functioning at school. In K. H. Rubin, W. M. Bukowski, \& B. Laursen (Eds.), Handbook of peer interactions, relationships, and groups (pp. 531-547). The Guilford Press. 